\title{
Modeling the Impact of Prehospital Triage on a True-Life Drip and Ship Mechanical Thrombectomy Urban Patient Cohort
}

\author{
Stavros Matsoukas ${ }^{a}$ Brian Giovanni ${ }^{a} \quad$ Liorah Rubinstein $^{a} \quad$ Shahram Majidia \\ Laura K. Stein ${ }^{b}$ Johanna T. Fifia, b \\ aDepartment of Neurosurgery, The Mount Sinai Hospital, New York, NY, USA; ${ }^{b}$ Department of Neurology, The Mount \\ Sinai Hospital, New York, NY, USA
}

\section{Keywords}

Large vessel occlusion · Stroke triage scale $\cdot$ Intravenous thrombolysis - Mothership cohort · Drip and ship cohort . Bypass model

\begin{abstract}
Objective: The aim of the study was to model the effect of prehospital triage of emergent large vessel occlusion (ELVO) to endovascular capable center (ECC) on the timing of thrombectomy and intravenous (IV) thrombolysis using real-world data from a multihospital system. Methods: We selected a cohort of 77 consecutive stroke patients who were brought by emergency medical services (EMS) to a nonendovascular capable center and then transferred to an ECC for mechanical thrombectomy (MT) ("actual" drip and ship [DS] cohort). We created a hypothetical scenario (bypass model [BM]), modeling transfer of the patients directly to an ECC, based on patients' initial EMS pickup address and closest ECC. Using another cohort of 73 consecutive patients, who were brought directly to an ECC by EMS and underwent endovascular intervention, we calculated mean door-to-needle and door-to-arterial puncture (AP) times ("actual" mothership [MS] cohort). Timings in the actual MS cohort and the actual DS cohort were compared to timings from the BM cohort.
\end{abstract}

Results: Median first medical contact (FMC) to IV thrombolysis time was $87.5 \mathrm{~min}$ (interquartile range $[\mathrm{IQR}]=38$ ) for the DS versus $78.5 \mathrm{~min}(\mathrm{IQR}=8.96)$ for the $\mathrm{BM}$ cohort, with $p=$ 0.1672. Median FMC to AP was $244 \mathrm{~min}(\mathrm{IQR}=97)$ versus 147 $\min (\mathrm{IQR}=8.96)(p<0.001)$, and median $\mathrm{FMC}$ to $\mathrm{TICI} 2 \mathrm{~B}+$ time was $299 \mathrm{~min}(\mathrm{IQR}=108.5)$ versus $197 \mathrm{~min}(\mathrm{IQR}=8.96)(p<$ 0.001) for the DS versus BM cohort, respectively. Conclusions: Modeled EMS prehospital triage of ELVO patients' results in shorter MT times without a change in thrombolysis times. As triage tools increase in sensitivity and specificity, EMS triage protocols stand to improve patient outcomes.

(C) 2021 The Author(s).

Published by S. Karger AG, Basel

\section{Introduction}

Since the advent of intravenous (IV) thrombolysis, patients with neurologic symptoms concerning for acute ischemic stroke have been brought by emergency medical services (EMS) to the nearest hospital designated as a stroke center. Patients undergo diagnostic imaging and, when appropriate, receive IV thrombolytics. Over the past decade, mechanical thrombectomy (MT) has become the standard of care for management of acute karger@karger.com www.karger.com/cee

Karger $\stackrel{\text { ' }}{5}$

GOPEN ACCESS (c) 2021 The Author(s).

Published by S. Karger AG, Basel

This is an Open Access article licensed under the Creative Commons Attribution-NonCommercial-4.0 International License (CC BY-NC) (http://www.karger.com/Services/OpenAccessLicense), applicable to the online version of the article only. Usage and distribution for commercial purposes requires written permission.
Correspondence to:

Johanna T. Fifi, johanna.fifi@mountsinai.org 
ischemic stroke caused by emergent large vessel occlusion (ELVO) [1]. For the purposes of this study, primary stroke centers are referred to as nonendovascular capable centers (nECCs), while thrombectomy capable centers and comprehensive stroke centers will be referred to as endovascular capable centers (ECCs). Once ELVO is diagnosed at a nECC, ELVO patients must be transferred to an ECC for MT. However, interhospital transfers have been associated with significant delays in treatment [2]. Earlier treatment times result in improved functional outcomes [3]. Prehospital triage protocols have been developed to improve prehospital recognition of ELVO and improve treatment times. During the period of this study, prehospital triage protocols were not being used by EMS personnel in New York City to identify ELVO patients who may be transported directly to ECCs.

The Mount Sinai Health System encompasses ECCs that receive patients from EMS and also receive transfers from multiple nECCs. The rate of stroke in the NYC EMS patient population is about 50 per 4,800 EMS calls (1\%) [4]. The percentage of acute stroke patients who received IV thrombolysis and MT changed from $7.8 \%$ to $8.4 \%$ and from $1.3 \%$ to $2.6 \%$ since MT became standard of care [5]. We have previously published that treatment times are significantly delayed for patients requiring interhospital transfer [6-8]. We hypothesized that treatment times would be shorter if patients are initially triaged to ECCs and sought to explore this in a modeled cohort.

\section{Methods}

\section{Data Collection}

We performed a retrospective analysis of a prospectively collected institutional quality improvement database. Study protocols for data collection and this analysis were approved by our Institutional Review Board. Recorded data points included first medical contact (FMC) times, EMS departure times, EMS pickup addresses, nECC and ECC arrival times, IV thrombolysis administration times, arterial puncture times, recanalization times, and patient outcomes.

\section{Patient Selection}

Between September 1, 2017, and March 31, 2019, 287 consecutive patients underwent MT within our health system. Patients with both anterior and posterior circulation occlusions were included in this analysis. Patients were further stratified into those who attended a nECC prior to transfer for MT ("drip and ship" [DS] cohort) and those who were brought directly to an ECC by EMS ("mothership" [MS] cohort). In order to limit our analysis to the impact of prehospital EMS logistics on treatment times, inpatients who experienced inhospital ischemic stroke and underwent MT were excluded. Patients whose records had incomplete EMS data were ex- cluded. Delayed decision-making regarding MT was also an exclusion factor. After these exclusions, 77 DS and $73 \mathrm{MS}$ patients were analyzed. Baseline patient characteristics are shown in Table 1.

\section{Modeling}

We created a hypothetical "bypass model" (BM) cohort, by calculating the "modeled" times of the DS patients in the case that they would have been transferred directly to an ECC instead of a nECC, with EMS triage. Using FMC as time zero, a care timeline was created for each patient. Within the BM cohort, patients' EMS pickup addresses were geocoded and mapped using Google Maps Application Programming Interface. The closest ECC was identified based on driving distance and driving times, and ambulance driving times were calculated using traffic data from the actual date and time of pickup, using Google Maps Application Programming Interface. However, since BM is a hypothetical cohort, times for the intrahospital events were calculated using mean times for door to thrombolysis, door to AP, and door to thrombolysis in cerebral infarction (TICI) $2 \mathrm{~B}+$ recanalization from the MS cohort. Therefore, the differences between BM and MS cohorts are as follows: $\mathrm{BM}$ is modeled based on real DS patients, while MS is actual patient data; FMC to ECC is calculated based on geocoding for the $\mathrm{BM}$ cohort, while it consists of real-life data for the MS cohort; door to thrombolysis, door to AP, and door to TICI 2B+ recanalization for the BM cohort are the relative calculated means from the MS cohort (we selected the means for these time metrics from the MS and not the DS cohort, since both BM and MS patients are supposed to receive thrombolysis in the ECC, while DS patients stop by in a nECC to receive thrombolysis and then get shifted to an ECC). We looked at the outcomes of time from FMC to thrombolysis, FMC to AP, and FMC to recanalization.

\section{Statistical Analysis}

We used SAS software to perform our analysis. Transport times followed a normal distribution, and we reported their means and standard deviations, while treatment times were not normally distributed; therefore, we reported their medians and interquartile ranges (IQRs). Student's $t$ test was conducted for comparing both the normally distributed and the non-normally distributed variables (since sample is $>30$ for the latter). We compared DS versus MS cohort and DS versus BM cohort. A $p$ value of $<0.05$ was considered as the level of statistical significance for this study.

\section{Results}

Baseline characteristics of the cohorts are reported in Table 1. Mean central hospital NIH stroke scale was 17 versus 16 for DS and MS cohorts. Respectively, the number of patients who received IV thrombolysis was 34 versus 31 (44.2\% vs. $42.5 \%)$, and pre-stroke modified Rankin score (mRS) was assessed as 0 in 42 versus 40 (54.5\% vs. $54.8 \%)$ patients, 1 in 10 versus 10 patients, 2 in 9 versus 5 patients, and 3 or greater in 16 versus 18 patients. The M1 segment of the middle cerebral artery was the most common site of occlusion for both cohorts. All comparisons are shown in Tables 2 and 3, with the corresponding $p$ 
Table 1. Baseline patient characteristics

\begin{tabular}{|c|c|c|}
\hline Baseline cohort characteristics & $\begin{array}{l}\text { DS } \\
(n=77)\end{array}$ & $\begin{array}{l}\text { MS } \\
(n=73)\end{array}$ \\
\hline \multicolumn{3}{|l|}{ Demographics and other information } \\
\hline Age, median (SD) & $73.4(13.2)$ & $72.8(13.2)$ \\
\hline Male sex, $n(\%)$ & $33(42.9)$ & $32(43.8)$ \\
\hline Female sex, $n(\%)$ & $44(57.1)$ & $41(56.2)$ \\
\hline Central hospital NIHSS, mean (SD) & $17(7)$ & $16(7)$ \\
\hline Thrombolysis, $n(\%)$ & $34(44.2)$ & $31(42.5)$ \\
\hline \multicolumn{3}{|l|}{ Medical history, $n(\%)$} \\
\hline Essential hypertension & $66(85.7)$ & $46(63)$ \\
\hline Atrial fibrillation & $37(48.1)$ & $29(39.7)$ \\
\hline Diabetes mellitus & $34(44.2)$ & $22(30.1)$ \\
\hline Hyperlipidemia & $22(28.6)$ & $26(35.6)$ \\
\hline Valvular heart disease & $3(3.9)$ & $0(0)$ \\
\hline Chronic kidney disease & $6(7.8)$ & $2(2.7)$ \\
\hline Ischemic stroke & $12(15.6)$ & $14(19.2)$ \\
\hline Transient ischemic attack & $5(6.5)$ & $2(2.7)$ \\
\hline Chronic heart failure & $8(10.4)$ & $6(8.2)$ \\
\hline Coronary artery disease or myocardial infarction & $13(16.9)$ & $11(15.1)$ \\
\hline Peripheral vascular disease & $4(5.2)$ & $4(5.5)$ \\
\hline \multicolumn{3}{|l|}{ Pre-stroke mRS, $n(\%)$} \\
\hline 0 & $42(54.5)$ & $40(54.8)$ \\
\hline 1 & $10(13)$ & $10(13.7)$ \\
\hline 2 & $9(11.7)$ & $5(6.8)$ \\
\hline$\geq 3$ & $16(20.8)$ & $18(24.7)$ \\
\hline \multicolumn{3}{|l|}{ Location of occlusion based on CTA, $n(\%)$} \\
\hline Internal carotid artery & $0(0)$ & $1(1.4)$ \\
\hline Internal carotid artery terminus & $16(20.8)$ & $12(16.4)$ \\
\hline Tandem occlusion & $2(2.6)$ & $4(5.5)$ \\
\hline Middle cerebral artery M1 & $36(46.8)$ & $31(42.5)$ \\
\hline Middle cerebral artery M2 & $13(16.9)$ & $19(26)$ \\
\hline Middle cerebral artery M3 & $1(1.3)$ & $1(1.4)$ \\
\hline Anterior cerebral artery & $0(0)$ & $1(1.4)$ \\
\hline Posterior circulation & $9(11.7)$ & $4(5.5)$ \\
\hline
\end{tabular}

DS, drip and ship; MS, mothership; mRS, modified Rankin score.

values. The mean EMS on-scene time for patients in the DS cohort was $21.3 \mathrm{~min}$. Individual on-scene times were used for the BM cohort in each patient. Scene to first hospital (either nECC or ECC) transport times for DS versus MS were $8.6 \mathrm{~min}(\mathrm{SD}=5)$ and $9.6 \mathrm{~min}(\mathrm{SD}=5)$, respectively $(p=0.13)$. Mean scene to first hospital transport times were $8.6 \mathrm{~min}(\mathrm{SD}=5)$ for DS versus $10.7 \mathrm{~min}$ $(\mathrm{SD}=3.7)$ for BM, $p=0.0001$ (Table 2). Mean FMC to ECC time was $211.2 \mathrm{~min}$ (85.5) for DS and $32 \mathrm{~min}$ (8.96) for $\mathrm{BM}(p<0.001)$. Mean total ambulance driving time, including the transfer, was $38.7 \mathrm{~min}$ (17.3) for DS and 10.7 min (3.68) for BM, $p<0.001$ (Table 2).

Similarly, median door to IV thrombolysis time was 57 $\min (I Q R=39.25)$ in DS model versus $46.5 \mathrm{~min}(\mathrm{IQR} N / \mathrm{A}$ here) in the $\mathrm{BM}(p=0.011)$, door to $\mathrm{AP}$ was $48 \mathrm{~min}(\mathrm{IQR}=$ $24)$ versus $115 \mathrm{~min}$ (IQR N/A here), $(p<0.001)$, and door

Bypassing PSC Decreases Time to

Treatment in the Urban Context to TICI $2 \mathrm{~B}+$ was $92 \mathrm{~min}(\mathrm{IQR}=49)$ versus $165 \mathrm{~min}(\mathrm{IQR}$ N/A here), $(p<0.001)$ for DS versus BM, respectively (Table 3). FMC to IV thrombolysis was $87.5 \mathrm{~min}(\mathrm{IQR}=38)$ versus $78.5 \mathrm{~min}(\mathrm{IQR}=8.96), p=0.1672$ (Table 3). Last, FMC to AP was $244 \mathrm{~min}(\mathrm{IQR}=97)$ versus $147 \mathrm{~min}$ $(\mathrm{IQR}=8.96)(p<0.001)$, and FMC to TICI $2 \mathrm{~B}+$ was $299 \mathrm{~min}$ $(\mathrm{IQR}=108.5)$ versus $197 \mathrm{~min}(\mathrm{IQR}=8.96)$ for $\mathrm{DS}$ versus $\mathrm{BM}$, respectively (Table 3 ). The aforementioned results are also graphically represented in Figure 1a, b.

\section{Discussion}

Using a real-life patient cohort from a stroke system of care within a large multihospital institution, we demonstrated that treatment times were shorter in a model of 
Table 2. Transport times (means with standard deviations, in minutes)

\begin{tabular}{llll}
\hline DS versus MS & DS & MS & $p$ value \\
\hline EMS on-scene time & $21.3(8.4)$ & $21.3(8.9)$ & 0.81 \\
Scene to first hospital & $8.6(5)$ & $9.6(5)$ & 0.13 \\
FMC to nECC & $29.9(10.8)$ & $\mathrm{N} / \mathrm{A}$ & - \\
Transfer & $30.1(15.9)$ & $\mathrm{N} / \mathrm{A}$ & - \\
FMC to ECC & $211.2(85.5)$ & $30.6(10.2)$ & $<0.001$ \\
Total ambulance & $38.7(17.3)$ & $9.6(5)$ & $<0.001$ \\
\hline DS versus bypass & $\mathrm{DS}$ & $\mathrm{BM}$ & $p$ value \\
\hline EMS on-scene time & $21.3(8.4)$ & $\mathrm{N} / \mathrm{A}$ & - \\
Scene to first hospital & $8.6(5)$ & $10.7(3.7)$ & 0.0001 \\
FMC to nECC & $29.9(10.8)$ & $\mathrm{N} / \mathrm{A}$ & - \\
Transfer & $30.1(15.9)$ & $\mathrm{N} / \mathrm{A}$ & - \\
FMC to ECC & $211.2(85.5)$ & $32.03(8.96)$ & $<0.001$ \\
Total ambulance & $38.7(17.3)$ & $10.74(3.68)$ & $<0.001$ \\
& & & \\
\hline
\end{tabular}

EMS, emergency medical services; FMC, first medical contact; $n E C C$, nonendovascular capable center; ECC, endovascular capable center; BM, bypass model; MS, mothership; DS, drip and ship.
Table 3. Treatment times (medians with IQRs, in minutes)

\begin{tabular}{lllc}
\hline DS versus MS & DS & MS & $p$ value \\
\hline Door to IV thrombolysis & $57(39.25)$ & $46.5(22.5)$ & 0.0465 \\
Door to AP & $48(24)$ & $115(53)$ & $<0.001$ \\
Door to TICl 2B+ & $92(49)$ & $165(83)$ & $<0.001$ \\
DIDO & $126(79)$ & N/A & - \\
FMC to IV thrombolysis & $87.5(38)$ & $79(18)$ & 0.12 \\
FMC to AP & $244(97)$ & $151(53.5)$ & $<0.001$ \\
FMC to TICl 2B+ & $299(108.5)$ & $201(83.5)$ & $<0.001$ \\
\hline DS versus bypass & DS & BM & $p$ value \\
\hline Door to IV thrombolysis & $57(39.25)$ & $46.5(\mathrm{~N} / \mathrm{A})$ & 0.0110 \\
Door to AP & $48(24)$ & $115(\mathrm{~N} / \mathrm{A})$ & $<0.001$ \\
Door to TICl 2B+ & $92(49)$ & $165(\mathrm{~N} / \mathrm{A})$ & $<0.001$ \\
DIDO & $126(79)$ & $\mathrm{N} / \mathrm{A}$ & - \\
FMC to IV thrombolysis & $87.5(38)$ & $78.5(8.96)$ & 0.1672 \\
FMC to AP & $244(97)$ & $147(8.96)$ & $<0.001$ \\
FMC to TICl 2B+ & $299(108.5)$ & $197(8.96)$ & $<0.001$ \\
\hline
\end{tabular}

IV, intravenous; AP, arterial puncture; $\mathrm{TICl}$, thrombolysis in cerebral infarction; DIDO, door in door out; FMC, first medical contact; BM, bypass model; MS, mothership; DS, drip and ship. prehospital triage with direct routing to ECCs, while preserving time to IV thrombolysis in an urban environment. These data add to evidence that triaging patients in the field will improve time to thrombectomy for ELVO patients and may result in improved outcomes, especially in a high-density population area. New York City is a densely populated city with high availability of nECCs and ECCs. Another bypass modeling study reported median actual EMS arrival to arterial puncture time of 215 min versus modeled bypass scenarios time to a STRATIS registry ECC of $127 \mathrm{~min}(p<0.001)$ and $121 \mathrm{~min}$ to the ideal ECC $(p<0.001)$ [9]. The difference in time reported in this study resulted in statistically significant differences in modeled 3-month disability [9]. When the patient is 
Fig. 1. a Graphical representation of FMC to IV thrombolysis, FMC to AP, and FMC to TICI $2 \mathrm{~B}+$ times for all cohorts. b Graphical representation of metrics, for all cohorts, as follows: scene to nECC, DIDO, nECC to ECC, scene to ECC, and door to TICI 2B+times. Door to TICI 2B+times are the same between MS and bypass cohorts. We created the hypothetical BM assuming that only transport times would be different compared to the MS cohort. Door to TICI $2 \mathrm{~B}+$ time in the $\mathrm{BM}$ group includes IV thrombolysis and therefore is longer compared to the DS door to TICI 2B+ time. FMC, first medical contact; IV, intravenous; AP, arterial puncture; TICI, thrombolysis in cerebral infarction; nECC, nonendovascular capable center; DIDO, door in door out; ECC, endovascular capable center; DS, drip and ship; BM, bypass model; MS, mothership.

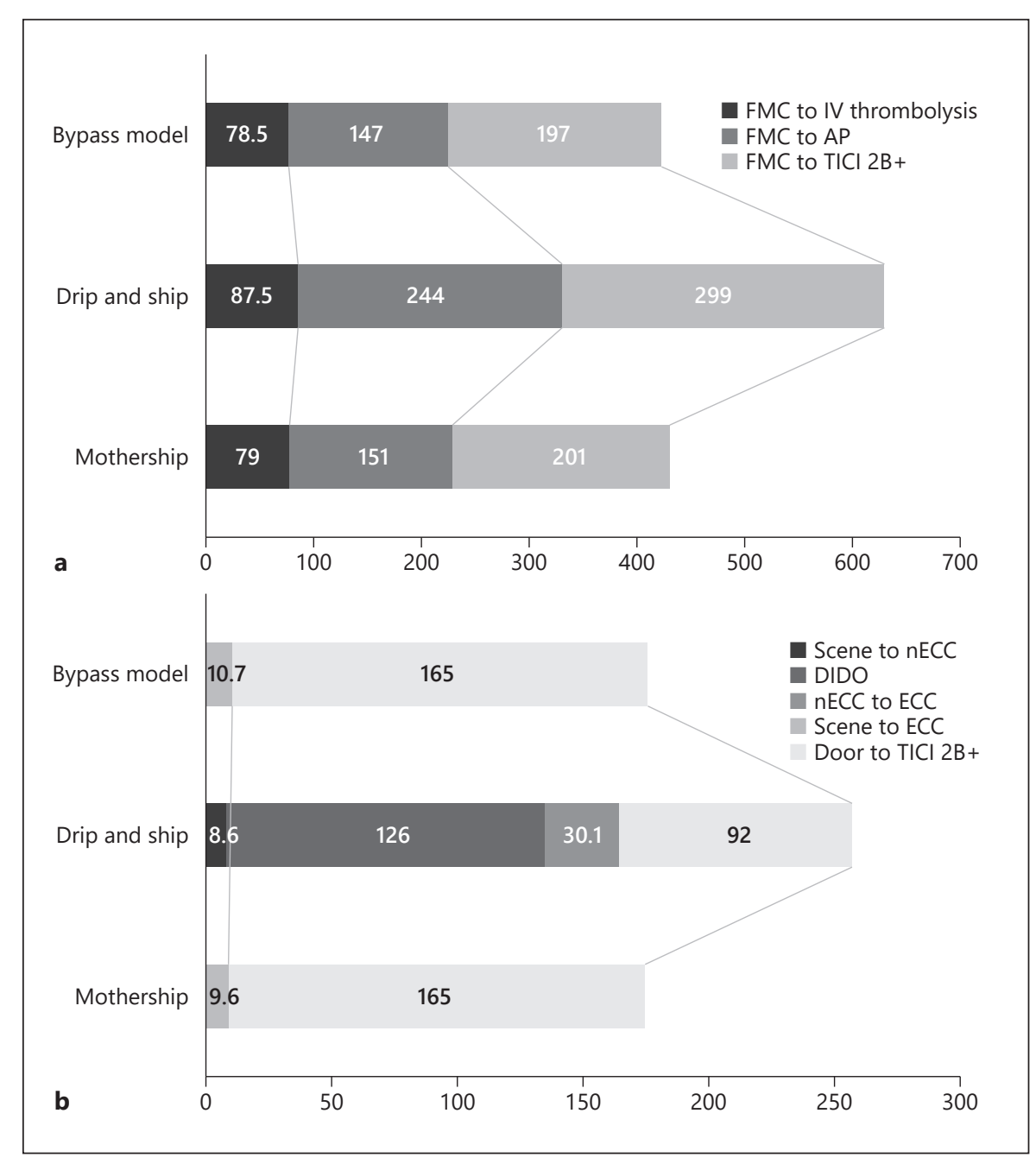

directly transferred to an ECC within 20 miles from the patient pickup point, MT can be achieved 94 min earlier, delaying thrombolysis by 7 minutes $[9,10]$, thus possibly ensuring eligibility for thrombolysis [9]. A recent metaanalysis including more than 2,000 patients from 9 studies reported better functional outcomes for patients treated via the MS versus the DS protocol (adjusted relative risk was $0.87,95 \%$ CI $0.77-0.98$, and unadjusted relative risk was $0.87,95 \% \mathrm{CI} 0.81-0.93, I^{2}=0.0 \%$ ). However, reperfusion success and 90-day mortality did not differ statistically significantly [11]. Notably, eligibility for MT can be decreased up to $41 \%$ when rerouting patients from a nECC to an ECC due to either clinical improvement after thrombolysis or changes in neuroimaging [2]. In addition, interhospital transfer has been associated with longer onset-to-AP and onset-to-recanalization times [12, 13]. Our study confirms that routing ELVO patients di- rectly to ECC centers should achieve shorter time final recanalization additionally, without the cost of delaying IV thrombolysis, in the urban setting.

Different mathematical models have been developed aiming to model treatment times of ELVOs based on geocoding and patient characteristics [14-17]. The prehospital transport decision-making is context-specific, and geographical distance from scene, transport times (based on map applications), treatment times at both nECC and ECC, as well as accuracy of available triaging tools have been advocated to be the most important determinants for such a decision [14, 17]. Milne et al. developed probability models based on the ESCAPE trial's decay curves in order to calculate and compare the probability of good outcomes (PGO) (90-day mRS score 0-2) between the DS and MS protocols. The authors concluded that when a nECC can achieve door-to-needle times 
less than $30 \mathrm{~min}$, DS is favored [15]. In continuation of this work, Holodinsky et al. [17] supported that DS and MS achieved similar modeled PGOs when the driving distance between the nECC and ECC was 60 min or less, by applying probability modeling. Similarly, Ali et al. [16] developed a mathematical model that provides locationbased optimal EMS routing choices using real-time data. Importantly, this model differs from previously done work, as it incorporates the possibility of different diagnoses, the diagnostic uncertainty of the triaging scales used at the field as well as the up-to-date performance of the different target hospitals. In contrast to the previously mentioned PGO studies, its output refers to cost and quality-adjusted life-years, thus providing a clinical instead of a probabilistic answer.

Utilization of screening scales for ELVO can result in more efficient patient workflow and achieve lower treatment times in both thrombolysis and endovascular treatments [18]. Already existing scales include the Los Angeles Motor Scale (LAMS), the Rapid Arterial Occlusion Evaluation (RACE), the Vision, Aphasia, Neglect, the Field Assessment Stroke Triage for Emergency Destination, the 3-step ambulance clinical triage for acute stroke treatment algorithm [19], and the Cincinnati Prehospital Stroke Severity Scale. It is important to note that a review of currently existing triage scales for ELVO supported that different scales may have a different sensitivity and/or specificity in different settings (field, emergency department) [20]. The Stockholm Stroke Triage System (SSTS), which includes a physical exam for hemiparesis, real-time screen by a nurse, and a telehealth assessment by a stroke expert, achieved 95\% specificity in both detecting ELVO and the need for MT [21]. In addition, specificity and overall accuracy were statistically significantly higher in the patient group that presented within $6 \mathrm{~h}$ after symptoms onset [21]. In a case-control study, triaging stroke patients with SSTS in the field resulted in faster MT times without delaying IV thrombolysis [22]. The RACE scale performed by EMS personnel has greater sensitivity compared to the SSTS scale, strong positive correlation with NIHSS score $(r=0.76)$ but poor specificity and positive predictive value [23]. Remarkably, specificity and positive predictive value were lower when RACE was implemented in stroke triage by US EMS personnel in a retrospective study [24]. The Cincinnati Prehospital Stroke Severity Scale did not differ statistically significantly from other currently used triaging systems in ELVO predictive performance [25]. In a prospective study, the use of LAMS proved superior compared to the conventional protocol of transferring patients to the nearest stroke hospital using geocoding [26]. Ultimately, this could lead in a substantially decreased number of secondary transfers [26]. False-negative results might include cases with good collateral circulation, in whom delays can be tolerated with less resultant morbidity [19]. On the other hand, hemorrhagic strokes that are falsely identified as ELVOs will still benefit if shifted to an ECC, where neurosurgical support is readily available [19]. Nevertheless, the exact impact of ELVO triaging scales has to be determined in large prospective trials. A new clinical triage scale for bypass, called SLAMS, is now being utilized by NYC EMS personnel in order to shift potential ELVOs to ECCs. The accuracy of this scale is not yet known.

Identifying MT-eligible patients in the field is a new challenge stemming from the ability to perform MT, while axial imaging serves as the gold standard method for identifying an ELVO. Technological improvements may further improve results. It has been reported that mobile stroke units could serve the role of primary stroke centers, administering IV thrombolysis and performing CT angiogram [27]. This, in conjunction with artificial intelligence, could expedite treatment and result in better functional outcomes. However, not every mobile stroke unit is capable of performing CT angiography, and it is not widely available in many cities. Therefore, clinical triage may be the only means for identifying ELVO patients at this current time. Importantly, these scales have a small learning curve and require minimal training. Triage tools for patients with ELVO in a densely populated area can potentially result in no delay in IV thrombolytic administration, with decreased onset-to-AP time and better 90-day outcomes [28]. The establishment and utilization of protocols that include early ECC notification and electronic platforms for instant image sharing can enhance treatment times and ensure better outcomes [29]. As far as limitations are concerned, our study tested a hypothetical model in a large city where there is proximity between nECCs and ECCs. Results may not be applicable to rural areas or in cities where these centers are sparsely dispersed on the map. In addition, our results may be markedly affected not only by geography, distances, travel times (this city has some of the highest densities of stroke units), weather, and traffic conditions, but also by the inhospital workflow of the individual hospital. Further work could evaluate the impact of triage scales on early and long-term clinical outcomes post-MT as these tools are being implemented in the real-life scenario. 


\section{Conclusion}

Based on our model, triaging patients with possible ELVO to the closest ECC is effective, resulting in earlier treatment times for ELVO patients compared to a DS cohort. Importantly, direct triage does not appear to sacrifice any valuable time in IV thrombolytics administration in the urban setting. This practice could potentially enhance long-term outcomes by affording faster treatment times with the proper implementation of triage tools by EMS.

\section{Statement of Ethics}

IRB approval was obtained by the Human Research Protection Program at the Icahn School of Medicine at Mount Sinai (ISMMS), and informed consent was waived due to the retrospective nature of the study (19-1975-00001-01).

\section{Conflict of Interest Statement}

The authors have no conflicts of interest to declare.

\section{Funding Sources}

This research work has received no grant from any funding agency in the public, commercial, or not-for-profit sectors.

\section{Author Contributions}

J.F. conceptualized the project. S. Matsoukas collected, analyzed, and interpreted the data, and drafted and significantly edited the manuscript and figures. All authors made final edits to the manuscript.

\section{Data Availability Statement}

Fully anonymized data are available and can be provided by the corresponding author, upon reasonable request.

\section{References}

1 Sweid A, Hammoud B, Ramesh S, Wong D, Alexander TD, Weinberg $\mathrm{JH}$, et al. Acute ischaemic stroke interventions: large vessel occlusion and beyond. Stroke Vasc Neurol. 2020;5(1):80-5.

2 Fuentes B, Alonso de Leciñana M, XiménezCarrillo A, Martínez-Sánchez P, Cruz-Culebras A, Zapata-Wainberg G, et al. Futile interhospital transfer for endovascular treatment in acute ischemic stroke: the madrid stroke network experience. Stroke. 2015 Aug;46(8): 2156-61.

3 Man S, Xian Y, Holmes DN, Matsouaka RA, Saver JL, Smith EE, et al. Association between thrombolytic door-to-needle time and 1-year mortality and readmission in patients with acute ischemic stroke. JAMA. 2020 Jun 2; 323(21):2170-84.

4 Brandler ES, Sharma M, McCullough F, BenEli D, Kaufman B, Khandelwal P, et al. Prehospital stroke identification: factors associated with diagnostic accuracy. J Stroke Cerebrovasc Dis. 2015 Sep;24(9):2161-6.

5 Stein L, Tuhrim S, Fifi J, Mocco J, Dhamoon M. National trends in endovascular therapy for acute ischemic stroke: utilization and outcomes. J Neurointerv Surg. 2020 Apr;12(4): 356-62.

6 Wei D, Oxley TJ, Nistal DA, Mascitelli JR, Wilson N, Stein L, et al. Mobile interventional stroke teams lead to faster treatment times for thrombectomy in large vessel occlusion. Stroke. 2017 Dec;48(12):3295-300.

7 Morey JR, Oxley TJ, Wei D, Kellner CP, Dangayach NS, Stein L, et al. Mobile interventional stroke team model improves early outcomes in large vessel occlusion stroke: the
NYC MIST trial. Stroke. 2020 Dec;51(12): 3495-503.

8 Morey JR, Zhang X, Marayati NF, Matsoukas $S$, Fiano E, Oxley T, et al. Mobile interventional stroke teams improve outcomes in the early time window for large vessel occlusion stroke. Stroke. 2021 Aug;52(9):e527-30.

9 Mueller-Kronast N, Froehler MT, Jahan R, Zaidat O, Liebeskind D, Saver JL. Impact of EMS bypass to endovascular capable hospitals: geospatial modeling analysis of the US STRATIS registry. J Neurointerv Surg. 2020 Nov;12(11):1058-63.

10 Froehler MT, Saver JL, Zaidat OO, Jahan R, Aziz-Sultan MA, Klucznik RP, et al. Interhospital transfer before thrombectomy is associated with delayed treatment and worse outcome in the STRATIS registry (systematic evaluation of patients treated with neurothrombectomy devices for acute ischemic stroke). Circulation. 2017 Dec 12;136(24):2311-21.

11 Ismail M, Armoiry X, Tau N, Zhu F, SadehGonik U, Piotin M, et al. Mothership versus drip and ship for thrombectomy in patients who had an acute stroke: a systematic review and meta-analysis. J Neurointerv Surg. 2019 Jan;11(1):14-9.

12 Gerschenfeld G, Muresan IP, Blanc R, Obadia M, Abrivard M, Piotin M, et al. Two paradigms for endovascular thrombectomy after intravenous thrombolysis for acute ischemic stroke. JAMA Neurol. 2017 May 1;74(5):54956.

13 Ciccone A, Berge E, Fischer U. Systematic review of organizational models for intra-arterial treatment of acute ischemic stroke. Int J Stroke. 2019 Jan;14(1):12-22.
14 Holodinsky JK, Williamson TS, Kamal N, Mayank D, Hill MD, GoyalDrip M, et al. Direct to comprehensive stroke center: conditional probability modeling. Stroke. 2017 Jan; 48(1):233-8.

15 Milne MS, Holodinsky JK, Hill MD, Nygren A, Qiu C, Goyal M, et al. Drip 'n ship versus mothership for endovascular treatment: modeling the best transportation options for optimal outcomes. Stroke. 2017 Mar;48(3): 791-4.

16 Ali A, Zachrison KS, Eschenfeldt PC, Schwamm LH, Hur C. Optimization of prehospital triage of patients with suspected ischemic stroke. Stroke. 2018 Oct;49(10): 2532-5.

17 Holodinsky JK, Williamson TS, Demchuk AM, Zhao H, Zhu L, Francis MJ, et al. Modeling stroke patient transport for all patients with suspected large-vessel occlusion. JAMA Neurol. 2018 Dec 1;75(12):1477-86.

18 Zaidi SF, Shawver J, Espinosa Morales A, Salahuddin H, Tietjen G, Lindstrom D, et al. Stroke care: initial data from a county-based bypass protocol for patients with acute stroke. J Neurointerv Surg. 2017 Jul;9(7):631-5.

19 Zhao H, Pesavento L, Coote S, Rodrigues E, Salvaris P, Smith K, et al. Ambulance clinical triage for acute stroke treatment: paramedic triage algorithm for large vessel occlusion. Stroke. 2018 Apr;49(4):945-51.

20 Zhelev Z, Walker G, Henschke N, Fridhandler J, Yip S. Prehospital stroke scales as screening tools for early identification of stroke and transient ischemic attack. Cochrane Database Syst Rev. 2019 Apr 9;4(4): Cd011427.
Bypassing PSC Decreases Time to Treatment in the Urban Context
Cerebrovasc Dis Extra 2021;11:137-144 DOI: $10.1159 / 000520078$ 
21 Almqvist T, Berglund A, Sjöstrand C, Eriksson E, Mazya MV. Prehospital triage accuracy in patients with stroke symptoms assessed within 6-24 hours or with an unknown time of onset. Stroke. 2021 Apr;52(4):1441-5.

22 Mazya MV, Berglund A, Ahmed N, von Euler M, Holmin S, Laska AC, et al. Implementation of a prehospital stroke triage system using symptom severity and teleconsultation in the Stockholm Stroke Triage Study. JAMA Neurol. 2020 Jun 1;77(6):691-9.

23 Pérez de la Ossa N, Carrera D, Gorchs M, Querol M, Millán M, Gomis M, et al. Design and validation of a prehospital stroke scale to predict large arterial occlusion: the rapid arterial occlusion evaluation scale. Stroke. 2014 Jan;45(1):87-91.
24 Dickson RL, Crowe RP, Patrick C, Crocker K, Aiken M, Adams A, et al. Performance of the RACE score for the prehospital identification of large vessel occlusion stroke in a suburban/ rural EMS service. Prehosp Emerg Care. 2019 Sep-Oct;23(5):612-8.

25 Crowe RP, Myers JB, Fernandez AR, Bourn S, McMullan JT. The cincinnati prehospital stroke scale compared to stroke severity tools for large vessel occlusion stroke prediction. Prehosp Emerg Care. 2021 Jan-Feb;25(1):6775.

26 Behnke S, Schlechtriemen T, Binder A, Bachhuber M, Becker M, Trauth B, et al. Effects of state-wide implementation of the Los Angeles Motor Scale for triage of stroke patients in clinical practice. Neurol Res Pract. 2021 Jun 1; $3(1): 31$.
27 Fassbender K, Grotta JC, Walter S, Grunwald IQ, Ragoschke-Schumm A, Saver JL. Mobile stroke units for prehospital thrombolysis, triage, and beyond: benefits and challenges. Lancet Neurol. 2017 Mar;16(3):227-37.

28 Jayaraman MV, Hemendinger ML, Baird GL, Yaghi S, Cutting S, Saad A, et al. Field triage for endovascular stroke therapy: a population-based comparison. J Neurointerv Surg. 2020 Mar;12(3):233-9.

29 McTaggart RA, Yaghi S, Cutting SM, Hemendinger M, Baird GL, Haas RA, et al. Association of a primary stroke center protocol for suspected stroke by large-vessel occlusion with efficiency of care and patient outcomes. JAMA Neurol. 2017 Jul 1;74(7):793800 